\section{Editorial}

J Prev Med Public Health 2019;52:71 • https://dx.doi.org/10.3961/jpmph.19.054

pISSN 1975-8375 elSSN 2233-4521

\title{
Our Valuable Contributors: Reviewers of 2018
}

I would like to recognize the peer reviewers who donated their precious time and effort to reviewing the manuscripts submitted to the Journal of Preventive Medicine and Public Health in 2018. With an ever increasing competition to attract quality research by major journals in our field, we depend on the expertise of the reviewers to critically appraise the papers. Their comments, detailed and extensive, guide authors to polish their work and propel them to strive for excellence. Their commitment and dedication to this hard and time consuming task ensure that published research are relevant, timely, high quality, and applicable.

I would like to extend my sincere gratitude to each and every one of the 118 reviewers.

Sung-il Cho, MD, ScD

Editor-in-Chief

Melanie Adams

Jeonghoon Ahn

Jisuk Bae

Jong-Myon Bae

Sang Geun Bae

Sanghyuk Bae

Hae-Kwan Cheong

Eunyoung Cho

Kyoung-Hee Cho

Sung Jun Cho

Sung-il Cho

Young-Gyu Cho

Ji-Yeop Choi

Kui Son Choi

Seongwoo Choi

Yong-jun Choi

Byung Cheol Chun

Jin-Ho Chun

Wankyo Chung

Rajabali Daroudi

Young Kyung Do

Sangyong Eom

Manal Etemadi

Sang Jun Eun

Dong-Hun Han

Young-Seoub Hong

Jeonghae Hwang

Tae Yoon Hwang

Yoko Ishihara

Abdolsaleh Jafari
Soong-Nang Jang

Sun Ha Jee

Boyoung Jeon

Keun-Ho Joe

Bak-Kuen Jung

Hye-Min Jung

Jaehun Jung

Minsoo Jung

Sun Jae Jung

Young-II Jung

Kyunghee Jungchoi

Moyeol Kang

Yunsik Kang

Ali Kazemi Karyani

Mohammad Khammarnia

Moran Ki

Myung Ki

Agnus M. Kim

Beomsoo Kim

Byoung Gwon Kim

Changhoon Kim

Changsu Kim

Gil-yong Kim

Hack-Lyoung Kim

Hwa Jung Kim

Hyeon Chang Kim

Hyoung-Ryoul Kim

Hyung Su Kim

Jong Yeon Kim

Jong-Hun Kim
Kyoung-Nam Kim

Mi Kyung Kim

Min Sun Kim

Rock Bum Kim

So Young Kim

Soon Duck Kim

Tae-Hyun Kim

Heejin Kimm

Kwang-Pil Ko

Sun Seog Kwon

Hee Young Lee

Hey Jean Lee

Hun Jai Lee

Joong-Yub Lee

Ju-Hyoung Lee

Jung-Eun Lee

Keon-Hyung Lee

Kwan Lee

Moo-Song Lee

Sunyoung Lee

Yo Han Lee

Young-Hoon Lee

Yu-Mi Lee

Hyunwoo Lim

Jungsu Lim

Roy Marzo

Mahmoud Moosazade

Jenyz Mundodan

Hae-Sung Nam

Naomi Noguchi
Minsu Ock

Changmo Oh

Gyung Jae Oh

In-Hwan Oh

Jin Kyoung Oh

Boyoung Park

Eun-Cheol Park

Jong Hyock Park

Kyoung Ok Park

Saengryeol Park

Sang Min Park

Sue Kyung Park

Sabarinah Prasetyo

Yahya Salimi

Aesun Shin

Hee-Young Shin

Ji-Yeon Shin

Min Ho Shin

Kyoung Hee Sohn

Hyunjin Son

Jee Hoon Son

Mia Son

Joohon Sung

Byongho Tchoe

Leila Vali

Charay Vichatahi

Pattaraporn Wongput

Seok-Ju Yoo 